\title{
Research on Sub Pixel Roundness Detection Technology Based on B-spline Lifting Wavelet
}

\author{
YanPing Gong \\ Electrical and mechanical Engineering College, \\ Institute of technology East China Jiaotong University \\ NanChang, China \\ e-mail: $83916971 @ q q . c o m$
}

\author{
ZhiGang Huang \\ School of mechatronic Engineering, \\ East China Jiaotong University \\ NanChang, China \\ e-mail: huangzg7301@163.com
}

\begin{abstract}
In order to realize roundness detection of rotary type parts in industrial field without interruption machining process, an image measurement method was proposed in this paper. Using adaptive threshold, the image edge can be determined by calculating modulus maximum in gradient direction in different scale. A single-pixel edge image will be obtained by synthesizing the multi-scale edge. Center drift problem in roundness evaluation had been solved by pole method. For improving efficiency and precision, a sub pixel roundness detection scheme was proposed by combining $B$ spline lifting wavelet with quadratic polynomial interpolation. The template part with known size was used to test the effects of different methods. The sub pixel technology can improve the resolution of image obviously. The experimental results show that the proposed detection method has better performance in terms of efficiency and accuracy compared with conventional detection methods. But the detection precision of proposed method was lower than that of roundness instrument measurement. There are still some work to be done in measuring method and denoising. For all that, the method can satisfy medium precision requirement in the general engineering application.
\end{abstract}

Keywords- roundness error; B-spline lifting wavelet; sub pixel; multi-resolution analysis; edge detection

\section{INTRODUCTION}

Roundness is an important form error for rotary type parts. It determines the part's machining accuracy, working accuracy and interchangeability. There exist three conventional measurement methods, i.e., characteristic parameter measurement, roundness instrument and image measurement. Based on two points' or three points' characteristic parameter in characteristic parameter measurement, the roundness error can be evaluated. This method has such merits as convenient measurement, simplicity. However, due to big error, this method is only fit for low precision situation. The roundness instrument measurement belongs to contact relative measurment. It is costly and is not fit for locale environment. The image measurement enlarges the actual profile of measured part by utilizing optical instrument. The roundness error can be evaluated by the enlarged profile image. This method has many merits such as low cost, high speed, high precision, easy integration and being fit for on-line detection. Because of the actual profile signal containing various frequency, the sampled signal must be filtered to extract roundness signal. B-spline lifting wavelet can effectively distinguish mutation and noise in signal used in this paper.
This wavelet has good filtering and denoising performence and can reconstruct the original signal. Therefore, the proposed method will make the roundness measurement and evaluation result more accuracy. Meanwhile, the computation complexity is the half of first-generation wavelet. That is to say, the real-time ability is greatly enhanced.

\section{MEASURING PRINCIPLE}

The measuring principle for roundness image measurement is shown as follows. CCD camera gathers the orthographic projection image of intermittent rotation part's side. The diameters in various cross sections within side image are calculated respectively. The two abovementioned steps will be repeated once the measured part revolves a certain angle. Based on the coordinate differences among many edge ultimate points in image, the evaluation of roundness error is accomplished[1]. Center drift problem in roundness measurement for diameter method has been solved by pole method in this paper.

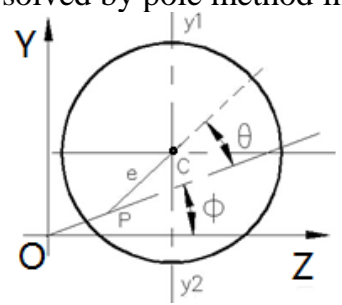

Figure 1. pole method measuring model

A measured cross-section is shown in Fig.1. YOZ is the camera coordinate. $\mathrm{P}$ is the rotation center of part. $\mathrm{C}$ is the geometry center of part. $\phi$ is the initial angle. $\theta$ is the measuring rotation angle. $\mathrm{e}$ is the eccentricity. $\mathrm{R}$ is the nominal diameter of part. $\delta \mathrm{r}(\phi)$ is the radial minimal variation. esin $(\theta+\phi)$ is the eccentric term. $y 1$ and $y 2$ are maximum and minimum edge coordinate in cross section, respectively.

$$
\begin{gathered}
y_{1}=e \sin (\theta+\phi)+r(\phi)+\mathrm{y}_{p} \\
=e \sin (\theta+\phi)+R+\delta r(\phi)+\mathrm{y}_{p} \\
y_{2}=e \sin (\theta+\phi)-r(\phi+\pi)+\mathrm{y}_{p} \\
=e \sin (\theta+\phi)-R-\delta r(\phi+\pi)+\mathrm{y}_{p}
\end{gathered}
$$

During the measured section having rotated a complete revolution, half value of the biggest difference in up and 
down edge variation is considered as the roundness error, i.e.

$$
\begin{gathered}
y_{1}-y_{2}=2 R+\delta r(\phi)+\delta r(\phi+\pi)=2 R+2 \delta r \\
f_{L S}=\max (\delta \mathrm{r})-\min (\delta \mathrm{r}) \\
=\frac{\max \left(y_{1}-y_{2}\right)-\min \left(y_{1}-y_{2}\right)}{2}
\end{gathered}
$$

\section{B-SPLINE LIFTING AlgORITHM AND Sub PIXEL EDGE DETECTION}

\section{A. B-spline Lifting Wavelet Algorithm}

Due to the base function being finite compactly supported,B-spline wavelet performance is superior to Gauss function. Therefore, the zero cross point and shape of spline curve can be controlled conveniently. B-spline function has such merits as good continuity, locality and noise suppression. As a smooth function, the B-spline has simpler filter coefficient and shorter length so as to reflect characteristics of the original image easily with smaller amount of calculation. The simulation was made in literature[2] for the B-spline function. It was proved that the order was larger, the closer fourier transform tended to ideal low-pass filter. When the B-spline function was third-order, its performance was approaching optimum. This showed that the local time-frequency performance of third-order B-spline function could satisfy most practical applications. Based on this point, the third-order B-spline function is adopted as smooth function for image edge detection in this paper.

Lifting wavelet decomposes signal into two non overlapping parts: odd subset and even subset. Every subset includes half sampling points of the original signal. Based on the correlation and appropriate prediction operator, signal value of a certain point can be obtained by adjacent signal values. The odd subset is usually predicted by the even subset[3]. Prediction error is the high frequency component in signal. The high frequency information in prediction stage uses update operator to adjust signal's down-sampling, i.e., the low frequency signal $\mathrm{c}$ which is gotten in equation (5). One important property of $c(n)$ is that its mean value shall be equal to the mean value of the original signal.

$$
\begin{aligned}
& d_{j-1}(n)=x_{j}(2 \mathrm{n}+1)-P\left[x_{j}(2 \mathrm{n})+x_{j}(2 \mathrm{n}+2)\right] \\
& c_{j-1}(n)=x_{j}(2 \mathrm{n})-U\left[d_{j-1}(\mathrm{n}-1)+d_{j-1}(\mathrm{n})\right]
\end{aligned}
$$

Inverse transformation is working backwards from the forward lifting operation. In the inverse step, the upate step is followed by predict step and finally the odd and even components are merged which interleaves the odd and even elements back into one data. The lifting algorithm is merely a new method to realize wavelet tranformation in essence. The signal property is determined by property of the adopted wavelet after wavelet transformation and is independent of the concrete scheme.

2D image is mostly handled in practical application. The two-dimensional function is generally decomposed by in-depth row-column analysis. That is to say, there are two steps. The first step is that the rows in gray-level matrix of image are transformed by lifting decomposition, and then, the results of first step are transformed by lifting decomposition according to column. Therefore, the original image is transformed into four sub-images after one decomposition, i.e., one is the low frequency subimage which reflects general picture characteristics of original image, the other three are high frequency subimages which reflect detail characteristics of original image along the horizontal, vertical and diagonal direction respectively, as shown in Fig.2.

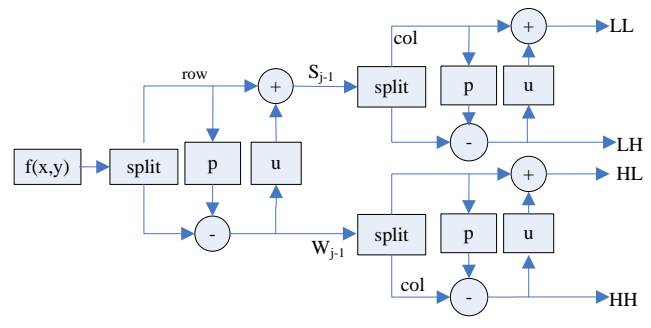

Figure 2. 2D lifting wavelet decomposition schematic diagram

$$
\left\{\begin{array}{l}
S_{2^{j}} f(\mathrm{n})=\sum_{k} h_{k} S_{2}{ }^{j} f\left(n-2^{j-1} k\right) \\
W_{2} f(\mathrm{n})=\sum g_{k} S_{2^{j-1}} f\left(n-2^{j-1} k\right), \mathrm{j}=1,2, \ldots \mathrm{J}-1
\end{array}\right.
$$

Where, $S_{2}{ }^{j} f(\mathrm{n})$ is general picture, $S_{2}{ }^{0} f(\mathrm{n})$ is original image , $W_{2} f(\mathrm{n})$ is detail image, $j$ is the current resolving scale, $J$ is the largest resolving scale, $h_{k}$ is low-pass filter, $g_{k}$ is high-pass filter. The real edge points can be detected in larger scale. Then, the positions of the real edge points can be determined accurately in smaller scale. The above mentioned strategy can improve the wavelet performance and enhance computational efficiency in image edge detection.

The lifting wavelet edge detection flow chart is shown in Fig.3. Firstly, the original image is smoothed by the third-order spline function. The result is the smooth function $\left(f^{*} \mathrm{Nj}\right)(\mathrm{n}, \mathrm{m})$.With regard to sub-image after decompostion, the wavelet ecofficiencies are calculated successively according to row and column. The local modulus maxima of smooth function along gradient direction is equal to the one of wavelet transformation. The module of gradient vector $\nabla(\mathrm{f} * \mathrm{Nj})(\mathrm{n}, \mathrm{m})$ is proportional to the one of wavelet transformation coefficiency as shown in equation (7). The module of wavelet coefficiency is calculated by equation (8) and then the largest modules in every direction are obtained. The foregoing modular image is partitioned based on relation between detection precision and pixel resolution in jth scale. Mean value of module in every partition is calculated to act as the threshold of each partition[4][5]. When module of a candidate edge point is larger than or equal to threshold, we can judge the point to be an edge point. On the contrary, the point will be deleted and its module value will be reset[6][7]. Considering that edge shift is less than one pixel between adjacent scales, a $3 \times 3$ matching area will be searched in potential edge image in (j-1)th scale according to an edge pixel in jth scale[8]. All 
potential edge points in the area are marked as candidate edge points. As a result, an candidate edge point iamge is gained in $(\mathrm{j}-1)$ th scale. Based on similarity condition between gradient and phase angle, the edge points are connected in current scale. By deleting isolated points, short chains and implementing edge thinning method introduced in literature[9], an edge image with single pixel can be produced. Owing to edge information in direction of diameter being needed in this measurement, the thinning work is only accomplished in left and right side of image. By virtue of edge image in jth scale and searching in $(j-1)$ th scale, a edge image in $(j-1)$ th scale is determined. After the recursion of above-mentioned process, the single pixel edge image of original image will be acquired eventually.
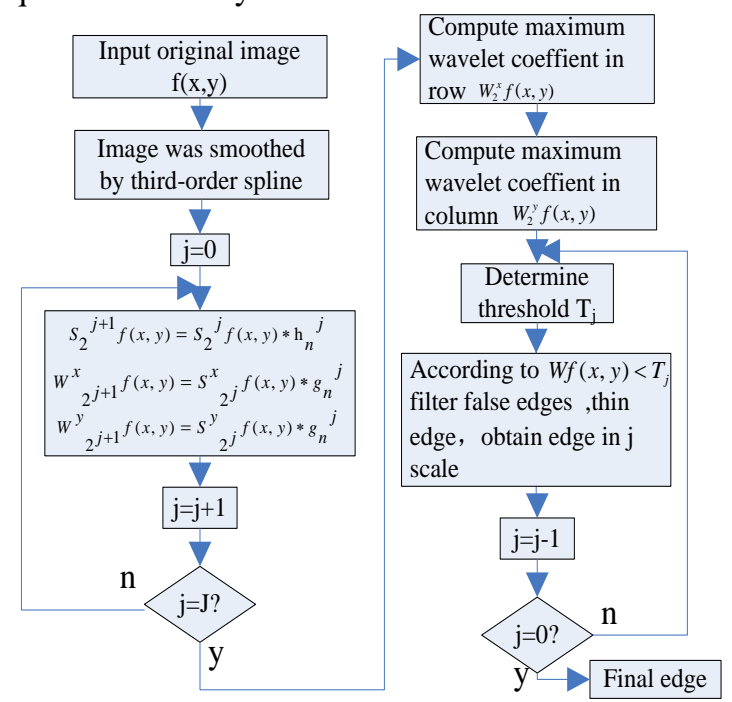

Figure 3. B-spline lifting wavelet edge detection flow chart

$$
\begin{aligned}
{\left[\begin{array}{c}
w_{j}^{1} f(n, m) \\
w_{j}^{2} f(n, m)
\end{array}\right] } & =j\left[\begin{array}{l}
\frac{\partial}{\partial x}\left(f * \mathrm{~N}_{j}\right)(\mathrm{n}, \mathrm{m}) \\
\frac{\partial}{\partial y}\left(f * \mathrm{~N}_{j}\right)(\mathrm{n}, \mathrm{m})
\end{array}\right]=j \nabla\left(f * \mathrm{~N}_{j}\right)(\mathrm{n}, \mathrm{m}) \\
M_{j} f(\mathrm{n}, \mathrm{m}) & =\sqrt{\left|w_{j}^{1} f(n, m)\right|^{2}+\left|w_{j}^{2} f(n, m)\right|^{2}}
\end{aligned}
$$

The phase angle, i.e., angle between gradient vector and horizontal direction is defined as follows.

$$
A_{j} f(\mathrm{n}, \mathrm{m})=\arctan \left[\frac{w_{j}^{2} f(n, m)}{w_{j}^{1} f(n, m)}\right]
$$

\section{B. Subpixel Edge Detection}

Image edge detection is the foundation and key in measurement. The edge obtained by existing edge detection algorithm has almost certain width. The positioning accuracy of this algorithm is in full-pixel with low detection resolution and disperse size. So, it is not suitable to apply this algorithm directly to precision measurement in practical application. It is analyzed theoretically that the biggest positioning error from pixel edge is 0.5 pixel. The number of pixels between two characteristic points may be close to one pixel error. The points which need sub pixel subdivision in image are not single isolated points but comprised of more than one point. These points have some geometric and gray distribution features which are basic requirements for sub pixel subdivision. The characteristic model can be established based on these information. Sub pixel positioning is divided into two steps, one is target recognition, the other is target positioning. The target recognition is defined to identify whether object measured is in the range of image. The target positioning is defined to compute target location which conforms to target properties by means of sub pixel detection. In this way pixel edge is divided into sub pixel edge[10].

In order to achieve sub pixel resolution, the interpolating function $\phi(x, y)$ based on $M(i, j)$ is obtained by bilateral gradient values of the largest gradient. And then, the maximum of $\phi(x, y)$ is computed. The coordinates of maximum point is the one of edge point. Three points is selected along $X$ direction such as $M(i-1, j)$, $M(i, j)$ and $M(i+1, j)$. Function $\phi(x)$ is the quadratic polynomial interpolation function of $\mathrm{M}(\mathrm{x})$ and it must be determined firstly. $\phi(\mathrm{x})$ is differentiated and the result is equal to zero. We can get the coordinate in $\mathrm{X}$ direction of derivative maximum point $\mathrm{Xe}$. Likewise, Three points is selected along $\mathrm{Y}$ direction such as $\mathrm{M}(\mathrm{i}, \mathrm{j}-1), \mathrm{M}(\mathrm{i}, \mathrm{j})$ and $\mathrm{M}(\mathrm{i}, \mathrm{j}+1)$. Function $\phi(y)$ is the quadratic polynomial interpolation function of $\mathrm{M}(\mathrm{y})$ and it must be determined firstly. $\phi(y)$ is differentiated and the result is equal to zero. We can get the coordinate in $\mathrm{Y}$ direction of derivative maximum point $\mathrm{Ye} . \mathrm{y}(\mathrm{x})$ is the interpolation function. $\mathrm{lj}(\mathrm{n})(\mathrm{x})$ is interpolation basis function. $f \mathrm{j}$ is the function value. $x i$ is interpolation node. In $\mathrm{X}$ direction, $\phi(\mathrm{x})$ is interpolation function, function values are $M(i-1, j), M(i, j)$ and $\mathrm{M}(\mathrm{i}+1, \mathrm{j})$, interpolation nodes are $\mathrm{xi}-\mathrm{W}, \mathrm{xi}$ and $\mathrm{xi}+\mathrm{W}$. In $\mathrm{Y}$ direction, $\phi(\mathrm{y})$ is interpolation function, function values are $\mathrm{M}(\mathrm{i}, \mathrm{j}-1), \mathrm{M}(\mathrm{i}, \mathrm{j})$ and $\mathrm{M}(\mathrm{i}, \mathrm{j}+1)$, interpolation nodes are yi-H, yi and $\mathrm{yi}+\mathrm{H}$.

$$
\begin{gathered}
\mathrm{y}(x)=\sum_{j=0}^{n} l_{j}^{(n)}(x) f_{j} \\
l_{j}^{(n)}(x)=\frac{\left(x-x_{0}\right) \ldots\left(x-x_{j-1}\right)\left(x-x_{\mathrm{j}+1}\right) \ldots\left(x-\mathrm{x}_{n}\right)}{\left(x_{j}-x_{0}\right) \ldots\left(x_{j}-x_{j-1}\right)\left(x_{j}-x_{\mathrm{j}+1}\right) \ldots\left(x_{j}-\mathrm{x}_{n}\right)}
\end{gathered}
$$

$W$ is the pixel-pitch in $X$ direction. $H$ is pixel-pitch in $Y$ direction. the above values are substituted in Eq (10). Let $d \phi(x) / d x=0$ and $d \phi(y) / d y=0$, the coordinates of edge point can be derived in $\mathrm{Eq}(12)$ and (13).

$$
\begin{gathered}
X_{e}=x_{i}+\frac{M(\mathrm{i}-1, \mathrm{j})-M(\mathrm{i}+1, \mathrm{j})}{M(\mathrm{i}-1, \mathrm{j})-2 M(\mathrm{i}, \mathrm{j})+M(\mathrm{i}+1, \mathrm{j})} \mathrm{g} \frac{W}{2} \cos \theta \\
Y_{e}=x_{i}+\frac{M(\mathrm{i}, \mathrm{j}-1)-M(\mathrm{i}, \mathrm{j}+1)}{M(\mathrm{i}, \mathrm{j}-1)-2 M(\mathrm{i}, \mathrm{j})+M(\mathrm{i}, \mathrm{j}+1)} \mathrm{g} \frac{H}{2} \sin \theta
\end{gathered}
$$

Where, $\theta$ is the angle between gradient direction of edge point and $\mathrm{x}$ positive direction.

\section{MEASURING EXPERIMENT}

\section{A. Measuring Process}

The part is clamped by conical nose of automatic centering clamp with external expansion wedge. The 
clamp consists of handspike, sleeve, claw valve bodies and spring as shown in Fig.4. The clamp is lower than the measuring platform. When the clamp rises to a certain height, the driving motor begins to make the measured part intermittent rotation. The camera takes sampling in predetermined rhythm. There are three valve bodies with flat circular arc at the top of sleeve as shown in Fig.4. Combine three valve bodies with sleeve to make a complete cylinder at the initial stage. The top of the handspike is cone-shape and can be matched with an inclined plane from oblate valve body. Thus, a doubleslider mechanism is formed. When there is no external force, the spring is in free state. During handspike moving upward under external force, the valve bodies will open outwards under the action of cone-shape inclined plane. At this moment the spring will be stretched. The part inner wall is swelled from inside out based on discontinuous cylindrical surface formed by three valve bodies. Through the above operation, part is clamped and positioned. When the external force is removed, valve bodies will return to start position under the action of elastic force. Therefore, the part is loosened. CCD camera shoots photograph every $15^{\circ}$. In this way, 24 photographs will be shot during part rotates a complete revolution. Due to the number of measured points is even number, each cross section is measured twice in the course of axlebox rotating a complete revolution. In order to enhance measuring accuracy, radial tiny variation $\delta \mathrm{r}(\theta \mathrm{i})$ can be defined to be the average of two measurements.

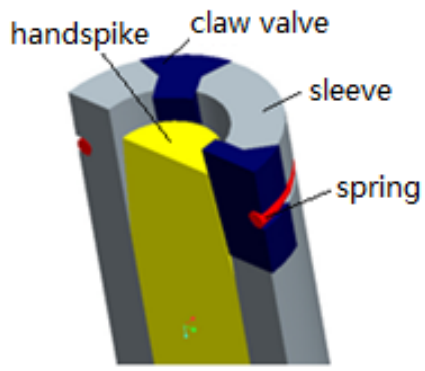

Figure 4. automatic centering clamp sectional view

\section{B. Experimental Result Analysis}

As a template, the dimensions of axlebox part with high precision must be basically the same as those of measured parts. The template part must be installed on the automatic centering clamp with external expansion wedge. The light source in back light board must be selected seriously. The array $C C D$ camera in this measuring system is CV-M4+CL. The resolution of camera is $1392 * 1040$. This image acquisition card labeled as $X 64$ CLIPRO is made by CORECO company from Canada. Standard parts with known parameters is used to calibrate. Based on sub pixel edge positioning and conversion, the calibration coefficent is $0.2339 \mathrm{~mm} / \mathrm{pixel}$. The detected binary edge images of axlebox part by means of Canny, Mallat wavelet and Lifting wavelet respectively are shown in Fig.4. The results showed that the edge detection effects of three operators are very similar and the lifting wavelet operator had the highest efficiency and shortest time. As can be seen from table 1, time consumptions of two methods had direct ratio relations with image size. It was obvious that the Lifting wavelet had faster speed in edge detection than the Mallat wavelet.

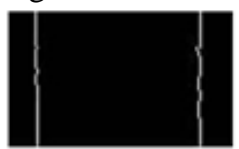

Canny

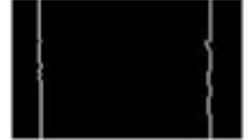

Mallat wavelet

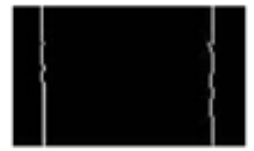

Lifting wavelet
Figure 5. The detected binary edge contour images using different operators

The data in Table 2 was obtained by sub pixel detection based on single pixel edge image in Fig.5, where the value of diameter was expressed by pixel. Due to the number of sampling point is even number, each diameter was measured twice in the course of axlebox rotating a complete revolution. Taking the arithmetic mean, 12 average diameters of the corresponding angle were obtained. The maximum of diameter is 68.535 pixel. The minimum of diameter is 67.968 pixel. The roundness error was equal to 0.2835 pixel based on Eq (3) and (4). After conversion, the roundness error of this part was equal to $0.066 \mathrm{~mm}$. The same part was measured by roundness instrument and the measuring value was $0.052 \mathrm{~mm}$. Results comparison: the value of roundness obtained in this measuring system was slightly larger than the one of roundness instrument. The reason was that the proposed method belonged to characteristic parameter measurement. Therefore, the error was an approximate roundness value. Nevertheless, this measuring system was competent for detection task in moderate accuracy situation.

\section{CONCLUSION}

The proposed method made full use of characteristics of lifting wavelet transformation in this paper. The edge of image was extracted in every scale using modified adaptive threshold. To obtain a precise single pixel edge, the multiscale edges must be synthesized. Based on quadratic polynomial interpolation function, the edge of image can be positioned precisely and sub pixel information of edge can be obtained simultaneously. In virtue of the above information of cross-section, the roundness measurement was accomplished. Experimental results showed that this method was feasible and had high precision as well as easy expansibility with low cost. These merits might realize the measuring process automatization, digitization and visualization so as to improve the detection efficiency greatly. At the same time, this method might provide reference to improve machining methods and process. The proposed method has carried on beneficial exploration and research for the development of part online detection. With the improvement of CCD technology, measuring precision as well as reduction of cost, there must be a better application prospect and wide space for this measuring method. 
TABLE I. THE COMPARISON OF TIME CONSUMPTION AMONG CANNY OPERATOR, MALLAT WAVELET AND LIFTING WAVELET

( UNIT: SECOND )

\begin{tabular}{c|c|c|c|c|c|c}
\hline $\begin{array}{c}\text { Same image with } \\
\text { different size }\end{array}$ & \multicolumn{3}{|c|}{$\begin{array}{c}\text { Edge in sub-image determined by first level } \\
\text { decomposition }\end{array}$} & \multicolumn{2}{c}{$\begin{array}{c}\text { Edge in sub-image determined by second } \\
\text { level decomposition }\end{array}$} \\
\cline { 2 - 7 } & Canny & Mallat & Lifting & Canny & Mallat & Lifting \\
\hline $128 * 128$ & 0.012 & 0.010 & 0.010 & 0.012 & 0.010 & 0.010 \\
\hline $256 * 256$ & 0.034 & 0.029 & 0.020 & 0.034 & 0.029 & 0.020 \\
\hline $512 * 512$ & 0.143 & 0.127 & 0.106 & 0.183 & 0.147 & 0.106 \\
\hline
\end{tabular}

TABLE II. SUB-PIXEL DIAMETEROF A CERTAIN CROSS SECTION AFTER 24 SAMPLING

\begin{tabular}{lcccccccc}
\hline rotation angle $\theta_{i}$ & $0^{0}$ & $15^{0}$ & $30^{0}$ & $45^{0}$ & $60^{0}$ & $75^{0}$ & $90^{0}$ & $105^{0}$ \\
\hline diameter $D_{i}$ (pixel) & 68.423 & 68.546 & 68.156 & 68.525 & 68.451 & 68.488 & 68.012 & 68.467 \\
rotation angle $\theta_{i}$ & $120^{0}$ & $135^{0}$ & $150^{0}$ & $165^{0}$ & $180^{0}$ & $195^{0}$ & $210^{0}$ & $225^{0}$ \\
diameter $D_{i}$ (pixel) & 68.563 & 68.187 & 68.339 & 68.387 & 68.542 & 68.439 & 67.993 & 68.545 \\
rotation angle $\theta_{i}$ & $240^{0}$ & $255^{0}$ & $270^{0}$ & $285^{0}$ & $300^{0}$ & $315^{0}$ & $330^{0}$ & $345^{0}$ \\
diameter $D_{i}$ (pixel) & 68.488 & 68.556 & 67.924 & 68.509 & 68.412 & 67.929 & 68.447 & 68.571 \\
\hline
\end{tabular}

\section{ACKNOWLEDGMENT}

This work was partially supported by a project from the Carrying Tool and Equipment Key Laboratory of Ministry of Education, East China Jiaotong University under Grant No. 12JD05, a project from Jiangxi province education department Grant No. GJJ14376.

\section{REFERENCES}

[1]Jing Min. A new measuring method of roundness error used in axial section image[J]. Manufacturing Automation,2014, 36(8):68-71.

[2]Lu Meng. Researching of Self-AdaptableWavelet Transform Arithmetic in the Image Edge Detection[D]. Shandong University,2011,4.

[3]G.M.Rajathi, Dr.R. Rangarajan. Performance Analysis of Image Denoising in Lifting Wavelet Transform $[\mathrm{J}]$. International Journal of Engineering Science and Technology, 2012,4(11): 4598-4602.
[4]WU Chang-dong, JIANG Hua, QIU Xiao-chu. Adaptive Threshould Based on Lifting Wavelet for Image Denoising[J].Microelectronics \& Coputer. 2010.27(12):18-21.

[5]Han Jing,Chen Youxing. Measurement methods of warhead scratch defect size based on adaptive threshold[J]. Journal of Applied Optics. 2013.34(5): 841-844.

[6]Chen Qiang,Sun Quansen,Xia Deshen.Serial Slice Image Segmentation of Digital Human Based on Adaptive Geometric Active Contour Tracking[J]. Computers in Biology and Medicine, 2013,43(6):635- 648.

[7]Manisha.s,Vandana c.Objective Evaluation Parameters of Image Segmentation Algorithms[J].International Journal of Engineering and Advanced Technology,2012, 2(2):84-87.

[8]Zhang Yumei.Research on Roundness on-Machine Measurement Based on multiscale analysis[D].Jilin University, 2009.

[9] Guo Lina. Sub-pixel Edge Detection of Color Images Based on Principal Axis[D]. Xi'an Technological

[10] Zhang Sijia.Study on Mechanical Precision Measurement Technology Based on Machine Vision[D].Shenyang University of Technology,2014. University, 2014. 